\title{
Cost-effectiveness of chronic kidney disease mass screening test in Japan
}

\author{
Masahide Kondo $\cdot$ Kunihiro Yamagata $\cdot$ Shu-Ling Hoshi $\cdot$ Chie Saito \\ Koichi Asahi $\cdot$ Toshiki Moriyama $\cdot$ Kazuhiko Tsuruya $\cdot$ Hideaki Yoshida $\cdot$ \\ Kunitoshi Iseki $\cdot$ Tsuyoshi Watanabe
}

Received: 6 October 2011/ Accepted: 11 November 2011/Published online: 14 December 2011

(C) The Author(s) 2011. This article is published with open access at Springerlink.com

\begin{abstract}
Background Chronic kidney disease (CKD) is a significant public health problem. Strategy for its early detection is still controversial. This study aims to assess the costeffectiveness of population strategy, i.e. mass screening, and Japan's health checkup reform.

Methods Cost-effectiveness analysis was carried out to compare test modalities in the context of reforming Japan's mandatory annual health checkup for adults. A decision tree and Markov model with societal perspective were constructed to compare dipstick test to check proteinuria only, serum creatinine (Cr) assay only, or both.

Results Incremental cost-effectiveness ratios (ICERs) of mass screening compared with do-nothing were calculated as $¥ 1,139,399 / \mathrm{QALY}$ (US $\$ 12,660 / \mathrm{QALY}$ ) for dipstick
\end{abstract}

On behalf of The Japanese Society of Nephrology Task Force for the Validation of Urine Examination as a Universal Screening.

M. Kondo $(\bowtie) \cdot$ S.-L. Hoshi

Department of Health Care Policy and Management, Graduate

School of Comprehensive Sciences, University of Tsukuba,

1-1-1 Tennoudai, Tsukuba, Ibaraki 305-8577, Japan

e-mail: mkondo@md.tsukuba.ac.jp

K. Yamagata $\cdot$ C. Saito

Department of Nephrology, Graduate School of Comprehensive

Human Sciences, University of Tsukuba, 1-1-1 Tennoudai,

Tsukuba, Ibaraki 305-8575, Japan

K. Asahi - T. Watanabe

Division of Nephrology and Hypertension, Fukushima Medical

University, 1 Hikariga-oka, Fukushima 960-1295, Japan

T. Moriyama

Health Care Center, Osaka University,

1-17 Machikaneyama-cho, Toyonaka, Osaka 560-0043, Japan test only, $¥ 8,122,492 / \mathrm{QALY}$ (US $\$ 90,250 / \mathrm{QALY}$ ) for serum $\mathrm{Cr}$ assay only and $¥ 8,235,431 / \mathrm{QALY}$ (US $\$ 91,505$ / QALY) for both. ICERs associated with the reform were calculated as $¥ 9,325,663 / \mathrm{QALY}$ (US $\$ 103,618 / \mathrm{QALY}$ ) for mandating serum $\mathrm{Cr}$ assay in addition to the currently used mandatory dipstick test, and $¥ 9,001,414$ /QALY (US $\$ 100$, 016/QALY) for mandating serum Cr assay and applying dipstick test at discretion.

Conclusions Taking a threshold to judge cost-effectiveness according to World Health Organization's recommendation, i.e. three times gross domestic product per capita of $¥ 11.5$ million/QALY (US \$128 thousand/QALY), a policy that mandates serum $\mathrm{Cr}$ assay is cost-effective. The choice of continuing the current policy which mandates dipstick test only is also cost-effective. Our results suggest that a population strategy for CKD detection such as mass screening using dipstick test and/or serum $\mathrm{Cr}$ assay can be justified as an efficient use of health care resources in a

\author{
K. Tsuruya \\ Department of Integrated Therapy for Chronic Kidney Disease, \\ Graduate School of Medical Sciences, Kyushu University, \\ 3-1-1 Maidashi, Higashi-ku, Fukuoka 812-8582, Japan \\ H. Yoshida \\ Second Department of Internal Medicine, Sapporo Medical \\ University School of Medicine, S-1, W-16, Chuo-Ku, \\ Sapporo, Hokkaido 060-8543, Japan \\ K. Iseki \\ Dialysis Unit, University Hospital of The Ryukyus, \\ 207 Uehara, Nishihara, Okinawa 903-0215, Japan
}


population with high prevalence of the disease such as in Japan and Asian countries.

Keywords Chronic kidney disease - Cost-effectiveness · Dipstick test $\cdot$ Mass screening $\cdot$ Proteinuria $\cdot$ Serum creatinine

\section{Introduction}

A consensus has been established that chronic kidney disease (CKD) is a worldwide public health problem [1, 2]. The effectiveness of its early detection and treatment to prevent progression to end-stage renal disease (ESRD) and premature death from cardiovascular disease has become widely accepted [3], while the strategy of its screening is still under debate [4]. Whereas high-risk strategies such as routine screening for diabetes patients and as a part of initial evaluation of hypertension patients are pursued in Western countries [5, 6], some argue that population strategies, such as mass screening, could be adopted in Asian countries where CKD prevalence is high [7].

Japan has a long history of mass screening programme for kidney diseases targeting school children and adults since the 1970s. Both urinalysis and measurement of serum creatinine $(\mathrm{Cr})$ level have been mandated to detect glomerulonephritis in annual health checkup provided by workplace and community for adults aged $\geq 40$ years old since 1992 [8]. However, glomerulonephritis was replaced as the leading cause of ESRD by diabetic nephropathy in 1998 , and the focus of mass screening policy for adults was shifted to control of lifestyle-related diseases. In 2008, the Japanese government launched a programme, Specific Health Checkup (SHC) and Specific Counselling Guidance, focusing on metabolic syndrome in order to control lifestyle-related diseases, targeting all adults between the ages of 40 and 74 years [9]. This is a combined programme of mass screening followed by health education or referral to physicians. During the process of this development of SHC, different types of screening test for kidney diseases were discussed in the health policy arena [10]. Abandonment of dipstick test to check proteinuria was initially proposed by the Ministry of Health, Labour and Welfare, which was opposed by nephrologists who emphasised the significance of CKD. As a consequence, serum $\mathrm{Cr}$ assay was alternatively dropped and dipstick test remained in the list of mandatory test items [11]. However, those found with proteinuria in SHC are not included in the health education programme nor referred to physicians in the following Specific Counselling Guidance that particularly targets metabolic syndrome. At the time, much attention was paid to a report from the USA which suggested the cost-ineffectiveness of mass screening for proteinuria [12], which encouraged the government to abandon dipstick test in their initial proposal.

From the viewpoint of CKD control, the current SHC and Specific Counselling Guidance are not adequate. Therefore, to present evidence regarding CKD screening test for the revision of SHC, which is due in 5 years from its start in 2008, the Japanese Society of Nephrology set up the Task Force for the Validation of Urine Examination as a Universal Screening. Since cost-effectiveness analysis provides crucial information for organising public health programmes such as mass screening, the task force conducted an economic evaluation as a part of their mission. This paper presents the value for money of CKD screening test demonstrated by the task force. The results have implications for CKD screening programmes not only in Japan but also for other populations with high prevalence of CKD such as in Asian countries.

\section{Methods}

We conducted cost-effectiveness analysis of CKD screening test in SHC with a decision tree and Markov modelling from societal perspective in Japan. In modelling, we carried out a deliberate literature survey to find the best available evidence from Japan, while reports from overseas were excluded. The PubMed database and Igaku Chuo Zasshi (Japana Centra Revuo Medicina), a Japanese medical literature database, were accessed with combinations of relevant terms such as CKD, health checkup etc. Additionally, we re-analysed our databases and carried out surveys where applicable.

\section{Participant cohort}

We assume that uptake of SHC does not change regardless of the choice of the test used for CKD screening, so we model a cohort of participants in SHC. Since the sex and age distribution of participants affects outcomes, we run our economic model by sex and age strata. Probabilities of falling into a sex and age stratum are adopted from a nationwide complete count report of SHC in 2008 [13]. Each value is shown in Table 1, and we estimate outcomes based on the prognosis of participants by initial renal function. We also run our economic model for 25 initial renal function strata defined by the combination of five levels of dipstick test results and five stages of CKD according to estimated glomerular filtration rate (eGFR) derived from serum $\mathrm{Cr}$ level. Probabilities of falling into an initial renal function stratum are calculated from the Japan Tokutei-Kenshin CKD Cohort 2008, which is a large cohort for the evaluation of SHC. Each value is shown in Table 1. 


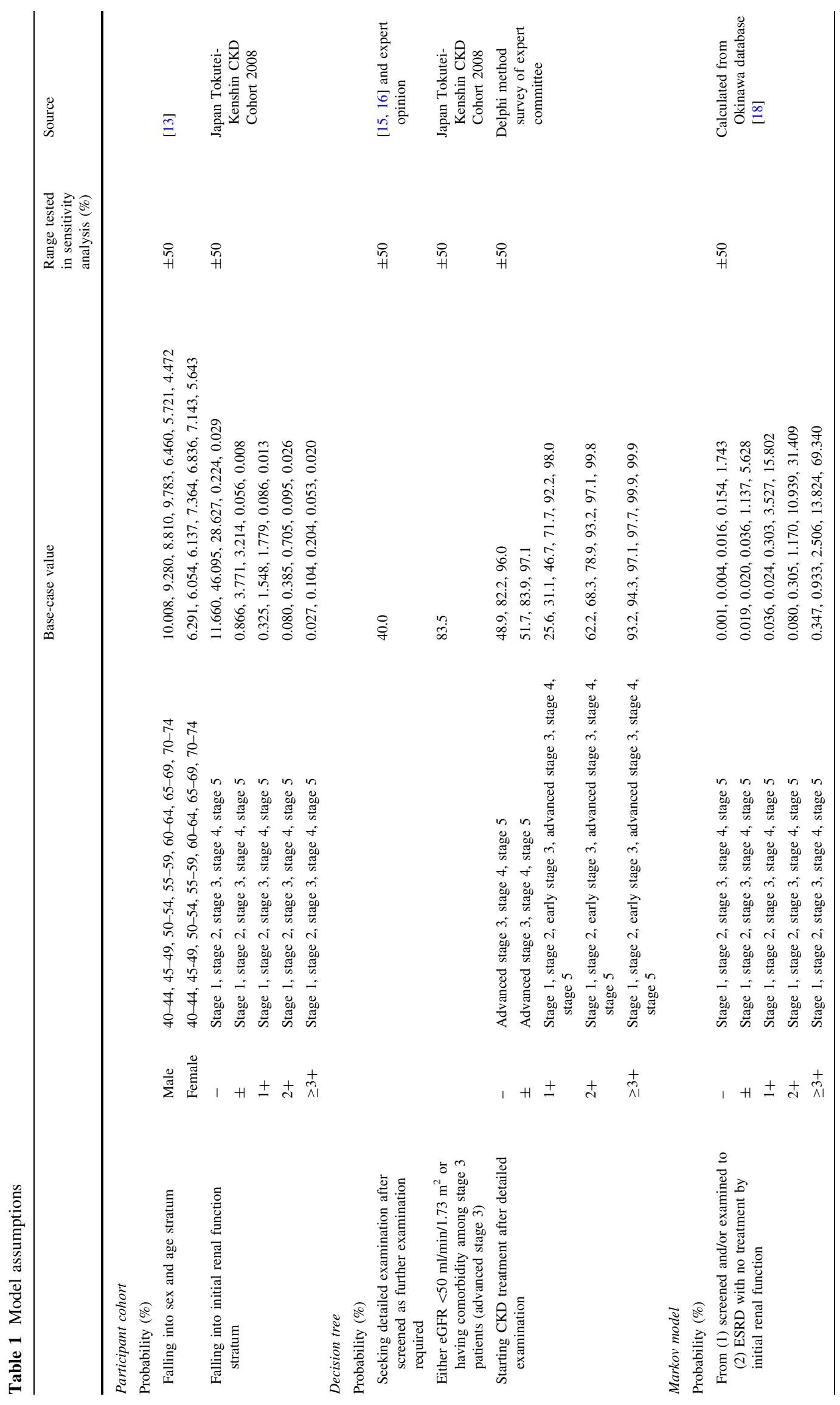




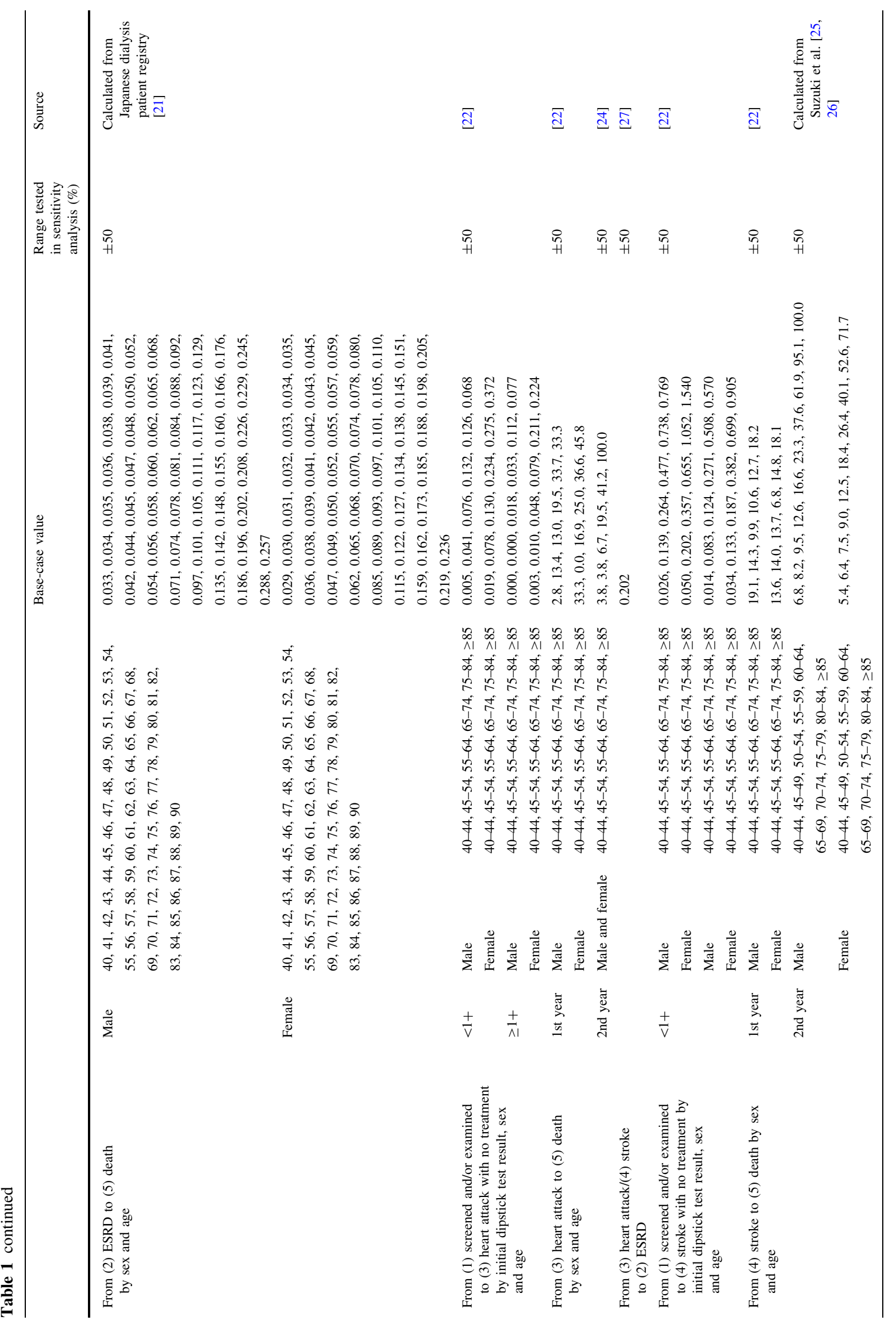




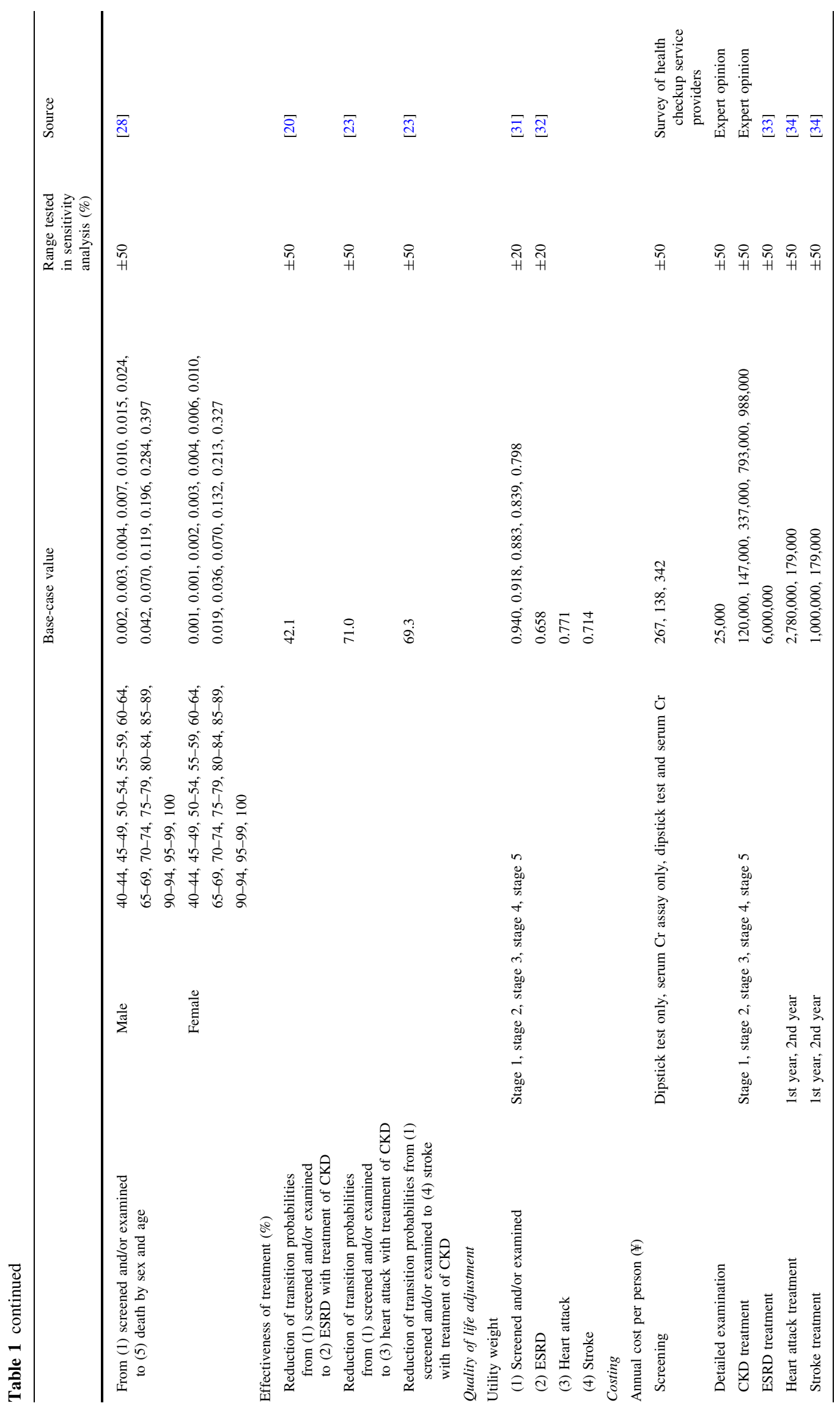




\section{Decision tree}

Figure 1a shows our decision tree comparing a do-nothing scenario with a screening scenario. After the decision node, participants under the do-nothing scenario follow the Markov model shown in Fig. 1b. For those under the screening scenario, three types of screening test are considered: (a) dipstick test to check proteinuria only, (b) serum $\mathrm{Cr}$ assay only and (c) dipstick test and serum $\mathrm{Cr}$ assay. Other tests such as microalbuminuria and cystatin $\mathrm{C}$ [14] are not considered, because they are not available options in the context of this study.

Screened participants are portioned between CKD patients who undergo treatment and those who are left untreated through three chance nodes. The first chance node divides the participants between those who require further examination and those left untreated. Participants with (a) dipstick test only, $\geq 1+$; with (b) serum $\mathrm{Cr}$ assay only, $\geq$ stage 3 ; and with (c) dipstick test and serum $\mathrm{Cr}$ assay, either $\geq 1+$ or $\geq$ stage 3 , are screened as requiring further examination. Those screened as requiring no further examination follow the Markov model. These are implemented by initial renal function stratum.

The second chance node divides participants screened as requiring further examination into those who seek detailed examination at health care providers and those who avoid any further examination. Its probability is assumed at $40.0 \%$ based on the literature $[15,16]$ and of the opinion of an expert committee set up for the purpose of this study, whose members are acknowledged in the "Acknowledgements" section. Those who avoid further examination follow the Markov model.

The third chance node divides participants who underwent further examination into those who undergo treatment of CKD and those left untreated. We derived these probabilities by initial renal function stratum with a Delphi survey of the expert committee. Regarding the strata of stage 3 CKD, a cut-off value of eGFR (50 ml/min/ $1.73 \mathrm{~m}^{2}$ ) and comorbidity such as hypertension, diabetes and/or hyperlipidaemia are considered in order to depict the difference in clinical practice when recommending start of treatment [17]. We label early stage 3 CKD and advanced stage $3 \mathrm{CKD}$ according to this criterion. Among stage 3 CKD patients, the probability of falling into advanced stage $3 \mathrm{CKD}$ by either eGFR $<50 \mathrm{ml} / \mathrm{min} /$ $1.73 \mathrm{~m}^{2}$ or having comorbidity is $83.5 \%$, calculated from the Japan Tokutei-Kenshin CKD Cohort 2008. Each value is shown in Table 1. All participants follow the Markov model after their completion of detailed examination.

\section{Markov model}

The Markov model consists of five health states: (1) screened and/or examined, (2) ESRD, (3) heart attack, (4) stroke and (5) death. Transitions between these states are indicated by arrows. Although individuals follow various courses other than these five health states and indicated transitions, we model in this way based on available data and literature.

We set the span of staying in each state of the Markov model at 1 year. Annual transition probabilities from (1) screened and/or examined to (2) ESRD with no treatment by the initial renal function stratum are calculated from our database of screened cohort in Okinawa Prefecture [18] for this study, since there is no operational predictive model for progression of CKD to ESRD such as Tangri et al. [19] in Japan. Each value is shown in Table 1. Reductions of these transition probabilities brought about by treatment of CKD
Fig. 1 Economic model. (M): Markov model
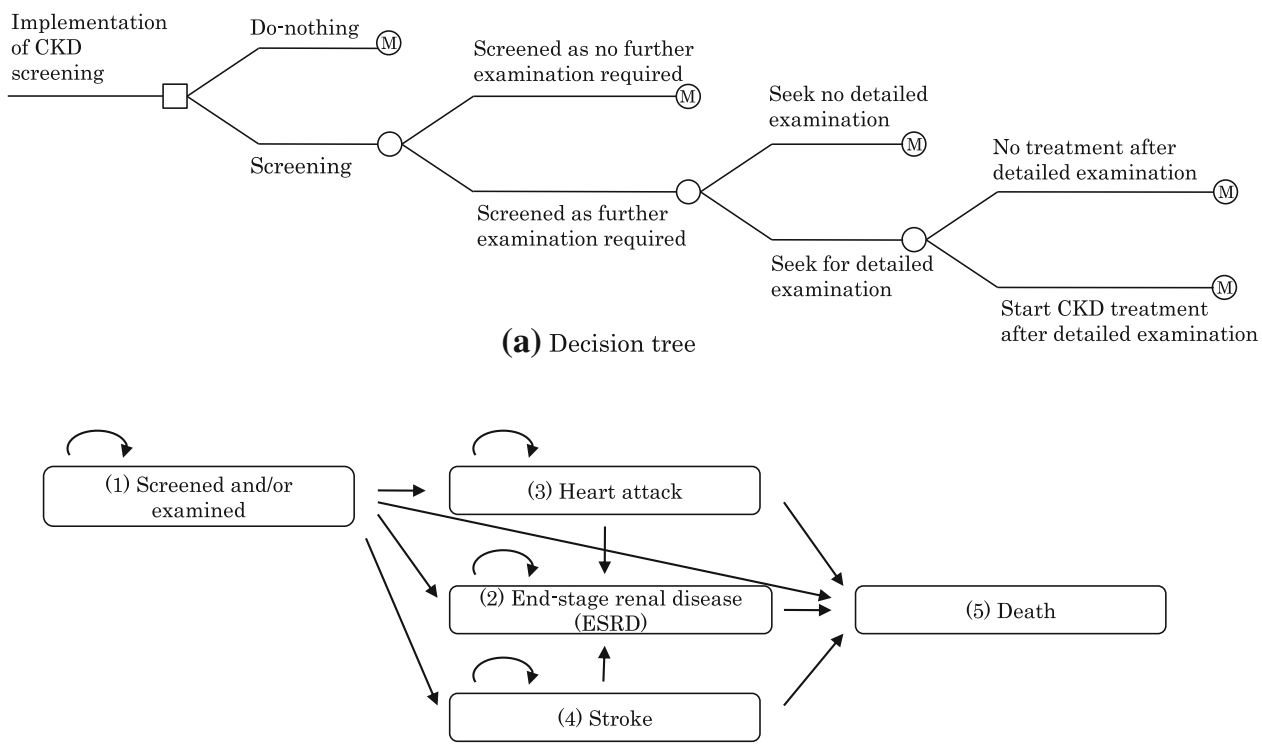

(b) Markov model 
are set at $42.1 \%$ based on Omae et al. [20], who investigated the effectiveness of angiotensin-converting enzyme inhibitor in improving renal prognosis. This is a unique Japanese evidence of treatment effectiveness evaluating progression to ESRD which can be compared with our Okinawa cohort [18]. The subsequent transition probabilities to (5) death are calculated from the life expectancy of dialysis starters according to a complete count report of Japanese patients on dialysis [21] by sex and age. Each value is shown in Table 1.

Transition probabilities from (1) screened and/or examined to (3) heart attack with no treatment are adopted from an epidemiological study in Okinawa by Kimura et al. [22] by initial dipstick test result, age and sex. Each value is shown in Table 1. Reductions of these transition probabilities brought about by treatment of CKD are set at $71.0 \%$ based on the Hisayama study by Arima et al. [23]. The subsequent transition probabilities to (5) death are adopted from Kimura et al. [22] by age and sex for the first year, and from Fukiyama et al. [24] for the second year and thereafter. Each value is shown in Table 1.

Transition probabilities from (1) screened and/or examined to (4) stroke with no treatment are adopted from Kimura et al. [22] by initial dipstick test result, age and sex. Each value is shown in Table 1. Reductions of these transition probabilities brought about by treatment of CKD are set at 69.3\% based on Arima et al. [23]. The subsequent transition probabilities to (5) death are adopted from Kimura et al. [22] by age and sex for the first year, and calculated from the Stroke Register in Akita of Suzuki [25, 26] for the second year and thereafter. Each value is shown in Table 1.

A transition probability from (3) heart attack and (4) stroke to (2) ESRD is adopted from an epidemiological study in Okinawa by Iseki et al. [27].

Transition probabilities from (1) screened and/or examined to (5) death are adopted from Vital Statistics of Japan 2008 [28] by age and sex. Each value is shown in Table 1.

We take a life-long time horizon so that the Markov cycle is repeated until each age stratum reaches 100 years old.

\section{Quality of life adjustment}

In order to estimate outcomes, use of quality-adjusted life years (QALYs) is recommended for economic evaluation of health care [29, 30]. QALYs are calculated as the sum of adjusted life-years experienced by a patient, where the adjustment is made by multiplying time by weights linked to the changing health state of the patient. The qualityadjustment weight is a value between 1 (perfect health) and 0 (death), which is one of the health-related quality of life measurements. Regarding (1) screened and/or examined, weights are assigned according to CKD stage based on initial renal function, using values adopted from Tajima et al. [31]. Weights for (2) ESRD, (3) heart attack and (4) stroke are cited from a past economic evaluation of antihypertensive treatment in Japanese context by Saito et al. [32].

\section{Costing}

From the societal perspective, costing should cover the opportunity cost borne by various economic entities in society. In the context of this study, costs borne by social insurers and patients are considered, since the cost of SHC is borne by social insurers and the cost of treatment is shared by social insurers and patients in Japan's health system. The amount of direct payments to health care providers by these entities is estimated as costs, while costs of sector other than health and productivity losses are left uncounted in this study. Cost items are identified along the decision tree and Markov model: screening, detailed examination, treatment of CKD, treatment of ESRD, treatment of heart attack and treatment of stroke. Each value is shown in Table 1.

Costs of screening were surveyed in five prefectures by inquiring health checkup service providers' price of adding CKD screening test to a test package that does not include renal function tests. Average price of those for (a) dipstick test to check proteinuria only, (b) serum $\mathrm{Cr}$ assay only and (c) dipstick test and serum $\mathrm{Cr}$ assay was $¥ 267$ (US $\$ 3.0$, with US \$1 = ¥90), ¥138 (US \$1.5) and ¥342 (US \$3.8) per person, respectively. Cost of detailed examination is set at $¥ 25,000$ (US \$278) per person according to the national medical care fee schedule and a treatment model developed by the expert committee. Annual costs of CKD treatment per person are set at $¥ 120,000$ (US $\$ 1,333$ ) for stage 1 CKD, $¥ 147,000$ (US $\$ 1,633$ ) for stage 2 CKD, $¥ 337,000$ (US $\$ 3,744$ ) for stage 3 CKD, $¥ 793,000$ (US $\$ 8,811$ ) for stage 4 CKD and $¥ 988,000$ (US $\$ 10,978$ ) for stage 5 CKD, also from the national medical care fee schedule and a treatment model developed by the expert committee. Annual cost of ESRD treatment per person, $¥ 6,000,000$ (US \$66,667), is cited from a review of renal disease care in Japan by Fukuhara et al. [33]. Annual cost of heart attack treatment per person, $¥ 2,780,000$ (US $\$ 30,889$ ) for the first year and $¥ 179,000$ (US $\$ 1,989$ ) for subsequent years, are cited from a past economic evaluation of cardiovascular disease prevention in Japanese context by Tsutani et al. [34]. Similarly, annual costs of stroke treatment per person, $¥ 1,000,000$ (US $\$ 11,111$ ) for the first year and $¥ 179,000$ (US $\$ 1,989$ ) for subsequent years, are cited from Tsutani et al. [34] as well.

\section{Discounting}

Both outcomes and costs are discounted at a rate of $3 \%$ [30]. 
Policy options for economic evaluation

To draw significant policy implications from this economic evaluation, policy options from status quo need to be defined. Under the current SHC, the dipstick test to check proteinuria is mandatory, while serum $\mathrm{Cr}$ assay is not. However, some health insurers voluntarily provide serum $\mathrm{Cr}$ assay to participants in addition to SHC. We surveyed health insurers in five prefectures and found that $65.4 \%$ of them implement use of serum $\mathrm{Cr}$ assay. Also, we analysed the Japan Tokutei-Kenshin CKD Cohort 2008 and found that $57.3 \%$ of participants underwent use of serum $\mathrm{Cr}$ assay. Therefore, we define the status quo regarding screening test for CKD as $40 \%$ of insurers implementing dipstick test only and $60 \%$ implementing dipstick test and serum $\mathrm{Cr}$ assay.

Then we evaluate two policy options in this study: 'Policy 1: Requiring serum $\mathrm{Cr}$ assay', and 'Policy 2: Requiring serum $\mathrm{Cr}$ assay and abandoning dipstick test'. Policy 1 means mandating use of serum $\mathrm{Cr}$ assay in addition to the currently used dipstick test, so that $100 \%$ of insurers implement both dipstick test and serum $\mathrm{Cr}$ assay if policy 1 is taken. Policy 2 is considered based on two recent health policy contexts. One is the discussion aroused during the development of $\mathrm{SHC}$ in which requiring serum $\mathrm{Cr}$ assay only and abandoning dipstick test used in the former occupational health checkup scheme attracted substantial support. It is expected that such a policy option will be proposed in the revision of SHC. Another relates to the change in diagnosis criterion of diabetes [35], in which a blood test to check the level of haemoglobin A1c instead of a dipstick test to check urinary sugar level has become pivotal. Implementing dipstick test for checking proteinuria only bears scrutiny from the viewpoint of economic evaluation. We assume that $100 \%$ of insurers would stop providing dipstick test if policy 2 is adopted.

We calculate incremental cost-effectiveness ratios (ICERs) for these two policy options using our economic model. ICER is a primary endpoint of cost-effectiveness analysis, which is defined as follows:

$$
\begin{aligned}
& \text { ICER }=\frac{\text { Incremental cost }}{\text { Incremental effectiveness }} \\
& =\frac{\text { Cost }_{\text {New policy }}-\text { Cost }_{\text {Status quo }}}{\text { Effectiveness }_{\text {New policy }}-\text { Effectiveness }_{\text {Status quo }}}
\end{aligned}
$$

This means the additional cost required to gain one more QALY under new policy.

\section{Sensitivity analysis}

Economic modelling is fundamentally an accumulation of assumptions adopted from diverse sources. Therefore, it is imperative to appraise the stability of the model. We perform one-way sensitivity analyses for our model assumptions. Assumed probabilities about the participant cohort, the decision tree and the Markov model are changed by $\pm 50 \%$. Reductions of transition probabilities brought about by treatment are also changed by $\pm 50 \%$. Utility weights for quality of life adjustments are changed by $\pm 20 \%$. Costs are changed by $\pm 50 \%$. Discount rate is changed from $0 \%$ to $5 \%$. We also changed our assumption about status quo that $40 \%$ of insurers implement dipstick test only and $60 \%$ implement dipstick test and serum $\mathrm{Cr}$ assay by $\pm 50 \%$ as well.

\section{Results}

Model estimators

Table 2 presents the model estimators. Under the do-nothing scenario, no patient is screened, with average cost of renal disease care per person of $¥ 2,125,490$ (US \$23,617) during average survival of 16.11639 QALY. When (a) dipstick test to check proteinuria only is applied, 832 patients out of 100,000 participants are screened, with additional cost of $¥ 7,288$ (US $\$ 81$ ) per person compared with the do-nothing scenario, for additional survival of 0.00639 QALY (2.332 quality-adjusted life days). When (b) serum $\mathrm{Cr}$ assay only is applied, 3,448 patients are screened with additional cost of $¥ 390,002$ (US \$4,333) per person compared with the do-nothing scenario, for additional survival of 0.04801 QALY (17.523 quality-adjusted life days). When (c) dipstick test and serum $\mathrm{Cr}$ assay are applied, 3,898 patients are screened with additional cost of $¥ 395,655$ (US \$4,396) per person compared with the donothing scenario, for additional survival of 0.04804 QALY (17.535 quality-adjusted life days).

Model estimators of ICERs were calculated as $¥ 1,139,399 /$ QALY (US \$12,660/QALY) for (a) dipstick test only, ¥8,122,492/QALY (US \$90,250/QALY) for (b) serum Cr assay only and $¥ 8,235,431 /$ QALY (US \$91,505/QALY) for (c) dipstick test and serum Cr assay.

\section{Cost-effectiveness}

Table 3 presents the results of cost-effectiveness analysis. Regarding the status quo that $40 \%$ of insurers implement dipstick test only and $60 \%$ implement dipstick test and serum $\mathrm{Cr}$ assay, 2,837 patients out of 100,000 participants are screened, with average cost of screening and renal disease care per person of $¥ 2,365,798$ (US \$212,922) during average survival of 16.14777 QALY. Taking policy 1 that $40 \%$ of insurers currently using dipstick test only start use of serum Cr assay screens more patients $(3,898)$. 
Table 2 Model estimators

\begin{tabular}{lcccccc}
\hline & $\begin{array}{l}\text { No. of patients } \\
\text { per 100,000 } \\
\text { participants }\end{array}$ & Cost (¥) & $\begin{array}{l}\text { Incremental } \\
\text { cost (¥) }\end{array}$ & $\begin{array}{l}\text { Effectiveness } \\
\text { (QALY) }\end{array}$ & $\begin{array}{l}\text { Incremental } \\
\text { effectiveness } \\
\text { (QALY) }\end{array}$ & $\begin{array}{l}\text { Incremental cost- } \\
\text { effectiveness ratio } \\
\text { (¥/QALY) }\end{array}$ \\
\hline Do-nothing & 0 & $2,125,490$ & & 16.11639 & \\
(a) Dipstick test only & 832 & $2,132,778$ & 7,288 & 16.12278 & 0.00639 & $1,139,399$ \\
(b) Serum Cr assay only & 3,448 & $2,515,492$ & 390,002 & 16.16440 & 0.04801 & $8,122,492$ \\
(c) Dipstick test and serum Cr assay & 3,898 & $2,521,145$ & 395,655 & 16.16443 & 0.04804 & $8,235,431$ \\
\hline
\end{tabular}

Table 3 Results of cost-effectiveness analysis

\begin{tabular}{|c|c|c|c|c|c|c|}
\hline & $\begin{array}{l}\text { No. of patients } \\
\text { per } 100,000 \\
\text { participants }\end{array}$ & Cost (¥) & $\begin{array}{l}\text { Incremental } \\
\text { cost (¥) }\end{array}$ & $\begin{array}{l}\text { Effectiveness } \\
\text { (QALY) }\end{array}$ & $\begin{array}{l}\text { Incremental } \\
\text { effectiveness } \\
(\text { QALY) }\end{array}$ & $\begin{array}{l}\text { Incremental cost- } \\
\text { effectiveness ratio } \\
\text { (¥/QALY) }\end{array}$ \\
\hline Status quo & 2,837 & $2,365,798$ & & 16.14777 & & \\
\hline Policy 1: requiring serum $\mathrm{Cr}$ assay & 3,898 & $2,521,145$ & 155,347 & 16.16443 & 0.01666 & $9,325,663$ \\
\hline $\begin{array}{l}\text { Policy 2: requiring serum } \mathrm{Cr} \text { assay } \\
\text { and abandoning dipstick test }\end{array}$ & 3,448 & $2,515,492$ & 149,694 & 16.16440 & 0.01663 & $9,001,414$ \\
\hline
\end{tabular}

It costs more, but it gains more. Its incremental cost is $¥ 155,347$ (US \$1,726), and its incremental effectiveness is 0.01666 QALY (6.081 quality-adjusted life days), resulting in ICER of $¥ 9,325,663 /$ QALY (US $\$ 103,618 /$ QALY). Taking policy 2 that $40 \%$ of insurers currently using dipstick test only start use of serum $\mathrm{Cr}$ assay and abandon dipstick test screens more patients $(3,448)$ compared with the status quo as well. It also costs more, but it gains more. Its incremental cost is $¥ 149,694$ (US \$1,663), and its incremental effectiveness is 0.01663 QALY (6.070 qualityadjusted life days), resulting in ICER of $¥ 9,001,414 /$ QALY (US \$100,016/QALY).

\section{Stability of cost-effectiveness}

One-way sensitivity analyses produce similar results not only between policy 1 and policy 2 but also among three model estimators of ICER. Therefore, we present a tornado diagram of policy 1 as an example in Fig. 2. Ten variables with large change of ICER are depicted. A threshold to judge cost-effectiveness is also drawn, which is according to World Health Organization's (WHO) recommendation, being three times gross domestic product (GDP) per capita [36]. Its value is $¥ 11.5$ million/QALY (US \$128 thousand/ QALY) gain in 2009 in Japan.

The effectiveness of CKD treatment to delay progression to ESRD is found to be the most sensitive. Decreasing the effect by $50 \%$ increases ICER to $¥ 16,280,537 /$ QALY (US $\$ 180,895 / \mathrm{Q} A L Y$ ). The effectiveness of CKD treatment to prevent stroke is also found to be the 10th largest change of ICER, but its range is limited.

The cost of treatment for stage 5 CKD is found to be the second most sensitive. Increasing the cost by $50 \%$ increases ICER to $¥ 14,404,335 / Q A L Y$ (US $\$ 160,048$ / QALY). The cost of ESRD treatment is found to be the fifth largest change, and the change is in the opposite direction; decreasing this increases ICER. Another cost item depicted is the cost of treatment for stage $3 \mathrm{CKD}$, which is found to be the sixth largest change.

The discount rate is found to be the third most sensitive. Discounting at a rate of $5 \%$ makes ICER $¥ 11,373,185$ / QALY (US \$126,369/QALY). Since policy 1 can screen CKD patients without proteinuria by use of serum $\mathrm{Cr}$ assay, the prognosis of non-proteinuric stage 5 CKD without treatment is found sensitive as the fourth and the seventh largest change. The eighth largest change depicted relates to the prevalence of CKD in participating population, i.e. stage $2 \mathrm{CKD}$ without proteinuria. The ninth largest change is utility weight for ESRD.

Taking the threshold to judge cost-effectiveness, oneway sensitivity analyses alter the interpretation of the results for only three variables: reductions of transition probabilities from (1) screened and/or examined to (2) ESRD with the treatment of CKD; cost of treatment for stage $5 \mathrm{CKD}$; and transition probability from (1) screened and/or examined to (2) ESRD with no treatment by initial renal function for stage $5 \mathrm{CKD}$ without proteinuria.

\section{Discussion}

We conduct a cost-effectiveness analysis of CKD screening test in SHC. Facing the scheduled revision of mandatory test items, we appraise two possible policy options compared with the status quo that $40 \%$ of insurers implement dipstick test to check proteinuria only and $60 \%$ implement 
Fig. 2 Tornado diagram of policy 1 . This tornado diagram shows ten variables which are found to be sensitive to the change in assumptions. Ten variables are presented, ordered according to the size of the change of ICER from top to bottom. The change of ICERs is represented by white bars when increasing the variable or by black bars when decreasing the variable from base-case value. The threshold to judge costeffectiveness is $3 \times$ GDP per capita (¥11.5 million/QALY gain)

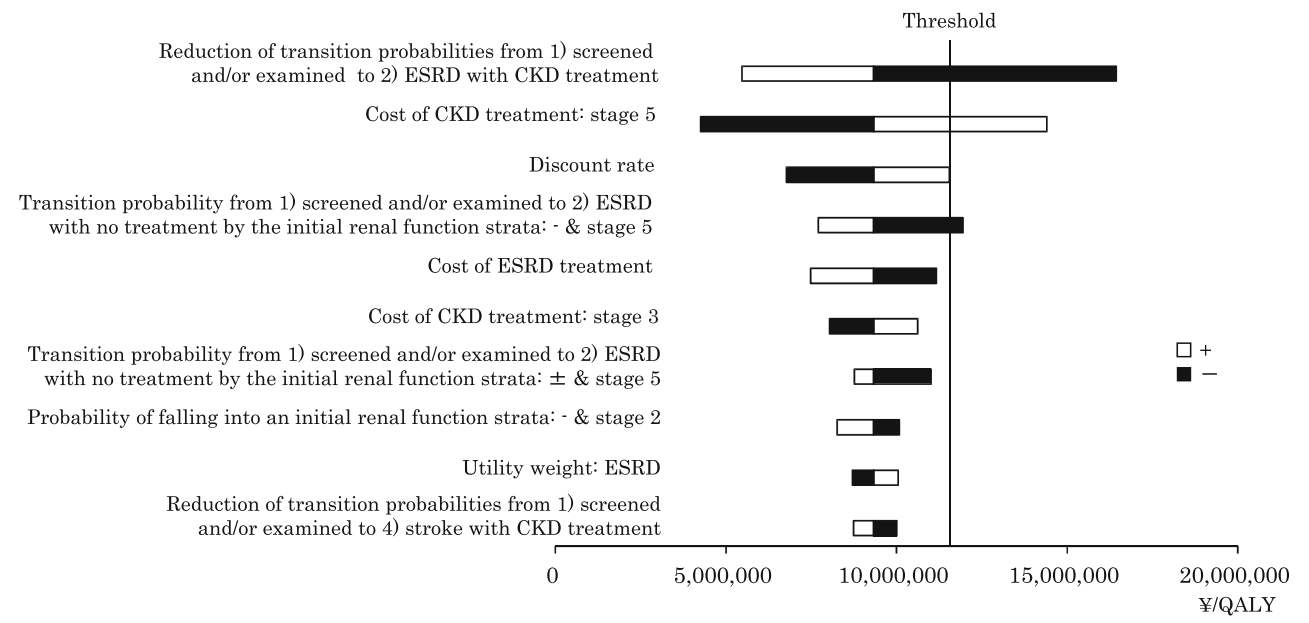

Reduction of transition probabilities from 1) screened 2) ESRD with CKD treatmen

Discount rate

ansition probability from 1) screened and/or examined to 2) ESRD Cost of ESRD treatment

Cost of CKD treatment: stage 3

withed to 2) ESRD with no treatment by the initial renal function strata: $\pm \&$ stage 5

Probability of falling into an initial renal function strata: - \& stage 2

Utility weight: ESRD

$5,000,000$ $¥ / \mathrm{QALY}$ dipstick test and serum $\mathrm{Cr}$ assay. Policy 1 is to mandate serum $\mathrm{Cr}$ assay in addition to the current dipstick test, so that $100 \%$ of insurers implement both dipstick test and serum $\mathrm{Cr}$ assay. Policy 2 is to mandate serum $\mathrm{Cr}$ assay and abandon dipstick test, so that $100 \%$ of insurers would stop providing dipstick test and switch to serum $\mathrm{Cr}$ assay. Our base-case analysis suggests that both policy options cost more and gain more. Estimated ICERs are $¥ 9,325,663$ / QALY (US \$103,618/QALY) for policy 1 and $¥ 9,001,414$ / QALY (US \$100,016/QALY) for policy 2.

To interpret these ICERs, there is no established value of social willingness to pay for one QALY gain in public health programmes such as mass screening in Japan, although some suggest $¥ 5$ million/QALY (US \$56 thousand/QALY) for an innovative medical intervention [37]. We follow WHO recommendation in this study, which is three times GDP per capita [36]. Its value is $¥ 11.5$ million/QALY (US $\$ 128$ thousand/QALY) gain in 2009 in Japan. Given this threshold, both policy 1 and policy 2 are judged as cost-effective. Therefore, mandating serum $\mathrm{Cr}$ assay in SHC can be justifiable as an efficient allocation of finite resources for health. Between policy 1 and policy 2, the ICER of policy 2 is slightly more favourable than that of policy 1 , while 450 more patients out of 100,000 participants are screened by adopting policy 1 . If secondary prevention of CKD is emphasised as a policy objective in addition to efficiency, policy 1 is an acceptable option as well as policy 2 .

Our model estimators have a policy implication, although estimated ICERs do not directly depict any marginal change in society. The ICER of (a) dipstick test only compared with the do-nothing scenario, ¥1,139,399/QALY (US \$12,660/QALY), is remarkably favourable. This implies that mass screening with dipstick test only is costeffective compared with abolishment of mass screening for kidney diseases altogether. Therefore, continuing the current policy, i.e. mandatory dipstick test, could be justifiable as an efficient resource allocation.
This contrasts with the reported cost-ineffectiveness of annual mass screening for adults using dipstick test to check proteinuria in the USA [12], although direct comparison cannot be made between the results of economic evaluations under different health systems. The difference could be attributable to the difference in the prevalence of proteinuria among screened population, with $5.450 \%$ being used in our model based on the Japan Tokutei-Kenshin CKD Cohort 2008, while $0.19 \%$ is assumed in the US study. Such epidemiological differences are known in terms of not only quantity but also in quality [7]. The prevalence of glomerulonephritis, especially $\operatorname{IgA}$ nephropathy, is higher in Asian countries including Japan compared with Western countries [10]. Also, the prevalence of renovascular disease such as ischaemic nephropathy, with which patients are often non-proteinuric until advanced stages of CKD, is lower in Asian countries [38]. The inclusion of heart attack and stroke into our model, which are excluded in the US model [12], may have also made the ICER more favourable.

There is a report of cost-ineffectiveness of populationbased screening for CKD with serum $\mathrm{Cr}$ assay from Canada [39]. This Canadian model can be compared with our model estimators of (b) serum $\mathrm{Cr}$ only compared with the do-nothing scenario. Their health outcomes gain or incremental effectiveness is 0.0044 QALY, which is smaller than ours, 0.04801 QALY, while their incremental cost is C \$463 (US \$441, using US $\$ 1=\mathrm{C} \$ 1.05$ ), which is also smaller than ours, $¥ 390,002$ (US $\$ 4,333$ ). These differences probably reflect the difference in the prevalence of CKD between Canada and Japan. Regarding the efficiency of screening programme, our model estimator of ICER, ¥8,122,492/ QALY (US \$90,250/QALY), is slightly more favourable than that of Canada, C \$104,900/QALY (US \$99,905/ QALY). However, the contradictory conclusion regarding cost-effectiveness is not due to this difference but rather the threshold taken. The Canadian study adopts lower value such 
as $\mathrm{C} \$ 20,000$ to $\mathrm{C} \$ 50,000 / \mathrm{QALY}$ (US $\$ 19,048$ to US \$47,619/QALY) following local practice [40].

Our sensitivity analysis suggests instability of the results in only three variables, so our findings are robust to a certain extent. The most sensitive variable is the effectiveness of CKD treatment delaying progression to ESRD: $42.1 \%$ reduction is adopted in our economic model according to the unique clinical evidence from Japan, whose agent is angiotensin-converting enzyme inhibitor. It is marginally larger than comparative values reported from Western countries. Reductions in the rate of GFR decline are $35.9 \%$ by Agodoa et al. [41], 39.8\% by The GISEN Group [42] and $22.5 \%$ by Ruggenenti et al. [43]. However, we think our assumption of base-case value is reasonable in two accounts: in light of the indication of angiotensin receptor blockers [17], whose use is more tolerated than angiotensin-converting enzyme inhibitors [44], and the higher prevalence of glomerulonephritis including IgA nephropathy, being a primary renal disease for ESRD, in Japan [10], for which the effect of early treatment such as renin-angiotensin system (RAS) inhibition, an immunosuppression, reduces risk of ESRD by $60 \%$ [45].

In regards to the other sensitive variables, we think the prognosis of non-proteinuric stage $5 \mathrm{CKD}$ without treatment does not greatly undermine our findings of base-case analysis, since the value is calculated from extended follow-up of an established database [18]. Uncertainty of the base-case value should be much less than the analysed $\pm 50 \%$. On the other hand, the cost of treatment for stage 5 CKD relates to one of the weaknesses of this study, as discussed in the following.

There are weaknesses in this study. The most significant one is that our economic model depicts the prognosis of CKD by initial renal function stratum. This approach is taken because of the limitation of epidemiological data, and it has little difficulty in estimating outcomes in terms of survival. However, it becomes problematic when it comes to costing. For example, a patient initially screened as stage 1 CKD stays at (1) screened and/or examined before transiting to the following health states such as (2) ESRD. This means that a patient skips over stage $2 \mathrm{CKD}$ to $5 \mathrm{CKD}$ before progressing to ESRD. To estimate the cost for this health state, the diversity of patients in terms of progression of the CKD stages should be taken into account. Our expert committee has developed treatment models to understand this problem. This type of uncertainty is larger in stage $1 \mathrm{CKD}$ and smaller in stage $5 \mathrm{CKD}$, but the cost of stages 1-4 CKD are not found to be so sensitive in our sensitivity analysis. Also, we think that uncertainty of the cost of stage $5 \mathrm{CKD}$, the second most sensitive variable, is less than the analysed $\pm 50 \%$, and our findings based on the base-case analysis are plausible. The problem
Table 4 Recommendation of the Japanese Society of Nephrology Task Force for the validation of urine examination as a universal screening

Mandate use of serum $\mathrm{Cr}$ assay in addition to the current dipstick test in the next revision of SHC

also affects quality of life adjustment, which tends to produce larger QALY outcomes.

Other weaknesses include our assumption of $100 \%$ adherence to treatment and so on. However, the most significant strength of this study is that our economic model depends totally on evidence from Japan only, which could justify our simplification in modelling on data availability basis. There is an opportunity for further refinement of our economic model, because a large-scale field trial evaluating the effect of multifactorial treatment including lifestyle modification for early-stage CKD [46] is ongoing in Japan, which will enable us to model progression of CKD with more rigorous clinical evidence [47].

In conclusion, we, the Japanese Society of Nephrology Task Force for the Validation of Urine Examination as a Universal Screening, recommend to mandate use of serum $\mathrm{Cr}$ assay in addition to the current dipstick test in the next revision of SHC, from the viewpoint of value for money and the importance of secondary prevention (Table 4). We think that continuation of current policy, in which dipstick test only is mandatory, is still a sensible policy option. Development of adequate Specific Counselling Guidance for screened participants is also recommended.

Whereas the primary objective of this study is to appraise policy options in Japanese context, it also demonstrates that good value for money can be expected from mass screening with dipstick test to check proteinuria in population with high prevalence; that is, a population strategy could be adopted for control of CKD. However, caution is needed when extrapolating this conclusion, since the scope of costing of our economic model does not cover the initial cost of launching mass screening. The model here is based on currently running SHC. The practice of annual mass screening for adults in Japan is quite exceptional, while such universal programmes are rarely found in other countries [48].

Acknowledgments We gratefully acknowledge contributions of the staff members who collected the data for this study at regional screening centres, Dr. T. Sairenchi for preparing the basic screening data, Ms M. Yokoyama for her assistance in medical cost calculation and Dr. S. Fujimoto, Dr. T. Konta, Dr. H. Sugiyama, Dr. N. Ura, Dr. Y. Yasuda, Dr. T. Tokura, Dr. E. Noiri, Dr. I. Narita and Dr. S. Uchida for their valuable discussions. This work was supported by Health and Labour Sciences Research Grants for "Research on the positioning of chronic kidney disease (CKD) in Specific Health Check and Guidance in Japan" (20230601), and a grant for strategic 
outcome study project for renal disease (H19-renal disease-senryaku001), the Ministry of Health, Labour and Welfare of Japan.

Conflict of interest The authors have declared that no conflicts of interest exist.

Open Access This article is distributed under the terms of the Creative Commons Attribution Noncommercial License which permits any noncommercial use, distribution, and reproduction in any medium, provided the original author(s) and source are credited.

\section{References}

1. Meguid El, Nahas A, Bello AK. Chronic kidney disease: the global challenge. Lancet. 2005;365:331-440.

2. Levey AS, Schoolwerth AC, Burrows NR, Williams DE, Stith $\mathrm{KR}, \mathrm{McClellan} \mathrm{W}$, et al. Comprehensive public health strategies for preventing the development, progression, and complications of CKD: report of an expert panel convened by the Centers for Disease Control and Prevention. Am J Kidney Dis. 2009;53:522-35.

3. Levey AS, de Jong PE, Coresh J, El Nahas M, Astor BC, Matsushita K, et al. The definition, classification and prognosis of chronic kidney disease: a KDIGO Controversies Conference report. Kidney Int. 2010;80:17-28.

4. Kiberd B. Screening for chronic kidney disease. BMJ. 2010;341:c5734.

5. de Jong PE, van der Velde M, Gansevoort RT, Zoccali C. Screening for chronic kidney disease: where does Europe go? Clin J Am Soc Nephrol. 2008;3:616-23.

6. Collins AJ, Vassalotti JA, Wang C, Li S, Gilbertson DT, Liu J, et al. Who should be targeted for CKD screening? Impact of diabetes, hypertension, and cardiovascular disease. Am J Kidney Dis. 2009;53:S71-7.

7. Chen N, Hsu CC, Yamagata K, Langham R. Challenging chronic kidney disease: experience from chronic kidney disease prevention programs in Shanghai, Japan, Taiwan and Australia. Nephrology (Carlton). 2010;15:31-6.

8. Imai E, Yamagata K, Iseki K, Iso H, Horio M, Mkino H, et al. Kidney disease screening program in Japan: history, outcome, and perspectives. Clin J Am Soc Nephrol. 2007;2:1360-6.

9. Kohro T, Furui Y, Mitsutake N, Fujii R, Morita H, Oku S, et al. The Japanese national health screening and intervention program aimed at preventing worsening of the metabolic syndrome. Int Heart J. 2008;49:193-203.

10. Yamagata K, Iseki K, Nitta K, Imai H, Iino Y, Matsuo S, et al. Chronic kidney disease perspectives in Japan and the importance of urinalysis screening. Clin Exp Nephrol. 2008;12:1-8.

11. Iseki K. Role of urinalysis in the diagnosis of chronic kidney disease (CKD). JMAJ. 2011;54:27-30.

12. Boulware LE, Jaar BG, Tarver-Carr ME, Brancati FL, Powe NR. Screening for proteinuria in US adults: a cost-effectiveness analysis. JAMA. 2003;290:3101-14.

13. Ministry of Health, Labour and Welfare. Heisei 20 nendo tokutei kenko shinsatokutei hoken shidono jisshi jyokyo ni tsuite. Tokyo: Ministry of Health, Labour and Welfare; 2010.

14. Peralta CA, Shlipak MG, Judd S, Cushman M, McClellan W, Zakai NA, et al. Detection of chronic kidney disease with creatinine, cystatin $\mathrm{C}$, and urine albumin-to-creatinine ratio and association with progression to end-stage renal disease and mortality. JAMA. 2011;305:1545-52.

15. AkamaY Y, Kikuchi S, Sato K, Okada T, Yamaguchi T. Shokuiki teiki kenko shindan ni okeru seimitsu kensa jushin jyokyo-dai chukibo jigyojyo to shokibo jigyojyo no hikaku. Sangyoeiseigaku Zasshi. 2006;48:S60-1.

16. Tsuda K, Tsutsumi A, Kawakami N. Work-related factors associated with visiting a doctor for a medical diagnosis after a worksite screening for diabetes mellitus in Japanese male employees. J Occup Health. 2004;46:374-81.

17. Japanese Society of Nephrology. Clinical practice guidebook for diagnosis and treatment of chronic kidney disease 2009. Tokyo: Tokyo Igakusha; 2009.

18. Iseki K, Iseki C, Ikemiya Y, Fukiyama K. Risk of developing end-stage renal disease in a cohort of mass screening. Kidney Int. 1996;49:800-5.

19. Tangri N, Stevens LA, Griffith J, Tighiouart H, Djurdjev O, Naimark D, et al. A predictive model for progression of chronic kidney disease to kidney failure. JAMA. 2011;305:1553-9.

20. Omae K, Ogawa T, Nitta K. Therapeutic advantage of angiotensin-converting enzyme inhibitors in patients with proteinuric chronic kidney disease. Heart Vessels. 2010;25:203-8.

21. Japanese Society for Dialysis Therapy. An overview of regular dialysis treatment in Japan as of 31 December , 2005. Tokyo: Japanese Society for Dialysis Therapy; 2006.

22. Kimura Y, Takishita S, Muratani H, Kinjo K, Shinzato Y, Muratani A, et al. Demographic study of first-ever stroke and acute myocardial infarction in Okinawa, Japan. Intern Med. 1998;37:736-45.

23. Arima H, Tanizaki Y, Kiyohara $\mathrm{Y}$, Tsuchihashi T, Kato I, Kubo M, et al. Validity of the JNC VI recommendations for the management of hypertension in a general population of Japanese elderly: the Hisayama study. Arch Intern Med. 2003;163:361-6.

24. Fukiyama K, Kimura Y, Wakugami K, Muratani H. Incidence and long-term prognosis of initial stroke and acute myocardial infarction in Okinawa, Japan. Hypertens Res. 2000;23:127-35.

25. Suzuki K. Stroke register in Akita: incidence and the burden of diseases. Nippon Ronen Igakkai Zasshi. 2008;45:169-71.

26. Suzuki K. Chiiki nosocchu hassho toroku wo riyo shita nosocchu iryo no shitu no hyoka ni kansuru kenkyu: Heisei 15 nendo-17 nendo sogo kenkyu hokokusho. Report of Health and Labour Sciences Research Grants (Contract No.: H16-KENKO-014). Tokyo: Ministry of Health, Labour, and Welfare; 2006.

27. Iseki K, Wakugami K, Maehara A, Tozawa M, Muratani H, Fukiyama K. Evidence for high incidence of end-stage renal disease in patients after stroke and acute myocardial infarction at age 60 or younger. Am J Kidney Dis. 2001;38:1235-9.

28. Ministry of Health, Labour and Welfare. Vital statistics of Japan 2008. Tokyo: Health and Welfare Statistics Association; 2010.

29. Drummond MF, Sculpher MJ, Torrance GW, O'Brien BJ, Stoddart GL. Methods for the economic evaluation of health care programmes. 3rd ed. Oxford: Oxford University Press; 2005.

30. Gold RM, Siegel JE, Russel LB, Weinstein MC. Cost-effectiveness in health and medicine. New York: Oxford University Press; 1996.

31. Tajima R, Kondo M, Kai H, Saito C, Okada M, Takahashi H, et al. Measurement of health-related quality of life in patients with chronic kidney disease in Japan with EuroQol (EQ-5D). Clin Exp Nephrol. 2010;14:340-8.

32. Saito I, Kobayashi M, Matsushita Y, Mori A, Kawasugi K, Saruta T. Cost-utility analysis of antihypertensive combination therapy in Japan by a Monte Carlo simulation model. Hypertens Res. 2008;31:1373-83.

33. Fukuhara S, Yamazaki C, Hayashino Y, Higashi T, Eichleay MA, Akiba $\mathrm{T}$, et al. The organization and financing of end-stage renal disease treatment in Japan. Int $\mathrm{J}$ Health Care Finance Econ. 2007;7:217-31.

34. Tsutani K, Igarashi A, Fujikawa K, Evers T, Kubin M, Lamotte $\mathrm{M}$, et al. A health economic evaluation of aspirin in the primary 
prevention of cardiovascular disease in Japan. Intern Med. 2007;46:157-62.

35. Seino Y. New diagnostic criteria for diabetes in Japan. Nippon Rinsho. 2010;68:2357-61.

36. Eichler HG, Kong SX, Gerth WC, Mavros P, Jönsson B. Use of cost-effectiveness analysis in health-care resource allocation decision-making: how are cost-effectiveness thresholds expected to emerge? Value Health. 2004;7:518-28.

37. Shiroiwa T, Sung YK, Fukuda T, Lang HC, Bae SC, Tsutani K. International survey on willingness-to-pay (WTP) for one additional QALY gained: what is the threshold of cost effectiveness? Health Econ. 2010;19:422-37.

38. Chrysochou C, Kalra PA. Epidemiology and natural history of atherosclerotic renovascular disease. Prog Cardiovasc Dis. 2009;52:184-95.

39. Manns B, Hemmelgarn B, Tonelli M, Au F, Chiasson TC, Dong $\mathrm{J}$, et al. Population based screening for chronic kidney disease: cost effectiveness study. BMJ. 2010;341:5869.

40. Menon D, Stafinski T. Health technology assessment in Canada: 20 years strong? Value Health. 2009;12:S14-9.

41. Agodoa LY, Appel L, Bakris GL, Beck G, Bourgoignie J, Briggs JP, et al. Effect of ramipril vs amlodipine on renal outcomes in hypertensive nephrosclerosis: a randomized controlled trial. JAMA. 2001;285:2719-28.

42. GISEN The Group (Gruppo Italiano di Studi Epidemiologici in Nefrologia). Randomised placebo-controlled trial of effect of ramipril on decline in glomerular filtration rate and risk of terminal renal failure in proteinuric, non-diabetic nephropathy. Lancet. 1997;349:1857-63.

43. Ruggenenti P, Perna A, Gherardi G, Garini G, Zoccali C, Salvadori M, et al. Renoprotective properties of ACE-inhibition in non-diabetic nephropathies with non-nephrotic proteinuria. Lancet. 1999;354:359-64.

44. Schmieder RE, Ruilope LM, Barnett AH. Renal protection with angiotensin receptor blockers: where do we stand. J Nephrol. 2011;24:569-80.

45. Hotta O, Miyazaki M, Furuta T, Tomioka S, Chiba S, Horigome I, et al. Tonsillectomy and steroid pulse therapy significantly impact on clinical remission in patients with IgA nephropathy. Am J Kidney Dis. 2001;38:736-43.

46. Gaede P, Lund-Andersen H, Parving HH, Pedersen O. Effect of a multifactorial intervention on mortality in type 2 diabetes. $\mathrm{N}$ Engl J Med. 2008;358:580-91.

47. Yamagata K, Makino H, Akizawa T, Iseki K, Itoh S, Kimura K, et al. Design and methods of a strategic outcome study for chronic kidney disease: frontier of renal outcome modifications in Japan. Clin Exp Nephrol. 2010;14:144-51.

48. Holland W. Screening for disease-consideration for policy. Euro Observer. 2006;8:1-4. 